\title{
Bevacizumab plus cisplatin and helical tomotherapy in treatment of locally advanced nasopharyngeal carcinoma
}

\author{
Jianxiong $\mathrm{Li}^{1, *}$ \\ Zhifei Zhao',* \\ Xuan $\mathrm{Wu}^{\mathrm{l}, *}$ \\ Jie $\mathrm{Yao}^{2, *}$ \\ Lin $\mathrm{Ma}^{\prime}$ \\ Rui Yel \\ Baolong Niu' \\ Lanqing Liang' \\ Xiao Zhao' \\ Qianqian Wang' \\ 'Radiology Department, Cancer \\ Center, The General Hospital \\ of the People's Liberation Army, \\ Beijing, People's Republic of \\ China; ${ }^{2}$ Department of Oncology, \\ The People's Liberation Army \\ I6I Hospital, Wuhan, People's \\ Republic of China \\ *These authors contributed equally \\ to this work
}

This article was published in the following Dove Press journal:

OncoTargets and Therapy

2 June 2015

Number of times this article has been viewed
Background: In recent years, chemotherapy, radiotherapy, and targeted drugs have been respectively used in nasopharyngeal carcinoma. In our research, we combined all of them for better curative effects.

Materials and methods: Thirty patients with histologically confirmed stage III IV (without distant metastasis) nasopharyngeal carcinoma were recruited between December 2013 and October 2014 with strict eligibility requirements and exclusion criteria. Bevacizumab $5 \mathrm{mg} / \mathrm{kg}$, $80 \mathrm{mg} / \mathrm{m}^{2}$ cisplatin and 67.5 Gy radiotherapy (helical tomotherapy [TOMO]) was given according to standard treatment protocols.

Results: After 3 months, 24 complete responses (80\%) and six partial responses (40\%) were observed in our research. Twenty-four patients $(80 \%)$ experienced oropharyngeal mucositis, $26 \%$ patients experienced weight loss caused by pharyngeal pain and dysphagia caused by oropharyngeal mucositis.

Conclusion: Bevacizumab plus cisplatin and TOMO in treatment of locally advanced nasopharyngeal carcinoma can increase curative effects with low side effects and toxicities.

Keywords: bevacizumab, cisplatin, helical tomotherapy (TOMO), nasopharyngeal carcinoma

\section{Introduction}

Nasopharyngeal carcinoma (NPC), also known as Guangdong carcinoma, endemic to Southern China is the most common malignant tumor of the head and neck. It is reported that about $80 \%$ NPC patients are in the People's Republic of China and almost $75 \%$ are at clinicopathologic stage III-IV (American Joint Committee on Cancer; sixth edition). ${ }^{1}$ The National Comprehensive Cancer Network Guidelines Version 2.2014 along with recent research recommends using a high-dose cisplatin and concurrent radiotherapy as the main treatment method for locoregionally advanced NPC. ${ }^{2-7}$ Therapeutic effects of conventional radiotherapy are generally limited due to various severe acute reactions. We replaced conventional radiotherapy with helical tomotherapy (TOMO) which has an advantage over intensity-modulated radiation therapy in better dose distribution, mild radiotherapy reaction, shorter single treatment time, and timely adjustment of setup error.

VEGF is a well-characterized protein with a central role in stimulating proliferation and angiogenesis, thus facilitating the formation and progression of human cancer. ${ }^{8} \mathrm{Sev}-$ eral groups have indicated that VEGF is aberrantly expressed in a broad range of cancers, including breast cancer, ${ }^{9}$ colorectal cancer, ${ }^{10}$ ovarian cancer, ${ }^{11}$ and NPC. ${ }^{12}$ Kyzas et al have reported that the high expression of VEGF leads to recurrence and death in NPC patients who received chemotherapy. ${ }^{13}$ Bevacizumab (Avastin) is the recombinant humanized monoclonal antibody against VEGF. Some trials have demonstrated that combining
The General Hospital of the People's Liberation Army, 28 Fuxing Road, Beijing 100853, People's Republic of China Email jianxiogl@।26.com 
bevacizumab with radiotherapy and cisplatin can increase progression-free survival with little toxic or side effects. ${ }^{14}$ There is an acknowledged hypothesis that the combination of bevacizumab with radiotherapy and cytotoxic agents may play a role in initially reducing tumor perfusion, vascular volume and microvascular volume. ${ }^{15}$ The widespread use of targeted drugs has changed the therapeutic strategy of treating NPC. Targeted drugs with concurrent radiochemotherapy can improve local control rate and 2-year progression-free survival. ${ }^{14,16}$ The primary purpose of this study was to evaluate the efficacy of concurrent radiochemotherapy with bevacizumab.

\section{Material and methods}

\section{Patient eligibility}

\section{Subjects}

The current research has been approved by the ethics committee of the General Hospital of the People's Liberation Army, Beijing, China. All patients provided written informed consent. The clinical trial has been approved by ClinicalTrials. gov (NCT02047487). Thirty patients with histologically confirmed stage III IV (without distant metastasis) NPC were recruited from People's Liberation Army general hospital between December 2013 and October 2014. Additional eligibility requirements included bi-dimensionally measurable primary tumor, Karnofsky Performance Scale $\geq 80$, survival expectancy $\geq 6$ months, good bone marrow function such as absolute neutrophil count $\geq 1,500 \mu / \mathrm{L}$, platelet count $\geq 100,000 \mu / \mathrm{L}$ and hemoglobin $\geq 9 \mathrm{~g} / \mathrm{dL}$. Patients were also required to have normal renal indicators including serum creatinine $\leq 1.5 \mathrm{mg} / \mathrm{dL}$ with or without creatinine clearance rate $\geq 55 \mathrm{~mL} / \mathrm{min}$. Furthermore, serum total bilirubin, glutamic oxalacetic transaminase, glutamic-pyruvic transaminase, and alkaline phosphatase should be no higher than 1.5 times the normal standard. Patients who were allergic to bevacizumab, and sustained dysbolism, serious illness (eg, alimentary tract hemorrhage, perforation), clinically significant heart disease, peripheral vascular disease, central nervous system dysfunction, an active infection $>$ National Cancer Institute Common Terminology Criteria for Adverse Events score 2, or pregnancy, serious liver disease (serum total bilirubin, glutamic oxalacetic transaminase, and glutamic-pyruvic transaminase $>2.5$ times the limit range of normal), serious kidney disease (urine protein $>1 \mathrm{~g} / 24 \mathrm{~h}$, glomerular filtration rate $<90 \mathrm{~mL} / \mathrm{min}$ ), or uncontrolled hypertension were excluded. We also excluded the patients taking aspirin or other anticoagulants.

\section{Drug therapy protocol}

Bevacizumab $5 \mathrm{mg} / \mathrm{kg}$ was given by intravenous infusion on day 1, 15, 29, and 43. Cisplatin was administrated at doses of $80 \mathrm{mg} / \mathrm{m}^{2}$ on days 2,23 , and 44 . Radiotherapy (helical tomotherapy [TOMO]) was given at the dose of $67.5 \mathrm{~Gy}$ from day 1 to the end. Chemotherapy was administered by giving hydration and diuresis for 3 days, and 5-hydroxytryptamine receptor antagonist was used prophylactically to prevent vomiting.

We terminated the cisplatin treatment if patients developed grade 3-4 toxicities and were unable to eat. The dosage was reduced by $25 \%$ when hematologic toxicities reached grade 3-4. Bevacizumab infusion was halted in case of hemorrhagic or thrombotic events.

\section{Radiotherapy protocol}

In helical TOMO, the gross tumor volumes (GTVs) at the primary site (GTVnx) and the gross tumor volume of the involved lymph nodes (GTVnd) included all disease volumes that were identified by computed tomography (CT), or magnetic resonance imaging (MRI). GTVnd1 and GTVnd2 can be recorded with more than one or two metastatic lymph nodes, respectively. The planning GTV site (pGTVnx) was designed to $3 \mathrm{~mm}$ margins around GTVnx. The real outspread distance depended on the actual distance between tumor and any of the following: brainstem, spinal cord, optic chiasma, or optic nerve. We divided target regions into two categories: clinical target volume (CTV)1 includes whole nasopharynx, skull base, clivus, parapharyngeal space, retropharyngeal lymph node region, sphenoid sinus, pterygopalatine fossa, nasal cavity, the back third of maxillary sinus, oropharynx, and level II and $\mathrm{V}$-a lymphatic drainage regions (including the upper cervical lymph nodes, level IB) using Brussels partition method. CTV1, CTV2 were designed to prevent CTV including level III, IV, V-a and V-b lymphatic drainage regions. Planning target volume (PTV)1 and PTV2 were defined as $3 \mathrm{~mm}$ margins around CTV1 and CTV2 and at least $3 \mathrm{~mm}$ below the skin surface. These lesions received $6 \mathrm{MeV}$ X-ray therapy, and 8-12 MeV electron beams. The prescription doses to pGTVnx, pGTVnd, PTV1, and PTV2 were 67.5 Gy/30 F, 67.5 Gy/3 0F, 60 Gy/30 F, and $54 \mathrm{~Gy} / 30 \mathrm{~F}$, respectively. Daily fraction of 2.25 Gy was delivered to the dose of tumor.

Clinical staging before therapy was assessed by pharyngorhinoscopy, MRI of nasopharynx and neck, chest CT, abdominal ultrasound, and bone scan. Adverse events were evaluated at weekly clinic visits using version 3.0 of the National Cancer Institute Common Terminology Criteria for Adverse Events. Radiologic assessment was performed by pharyngorhinoscopy and MRI of nasopharynx and neck 12 weeks after radiation therapy. 


\section{Endpoints and statistical analysis}

Overall response rate was calculated after 3 months among patients with local advanced NPC receiving concurrent cisplatin chemotherapy treatment. TOMO and bevacizumab toxicity to skin, mucosa and sialaden was evaluated by the Radiation Therapy Oncology Group during radiotherapy. Systemic toxicity was graded according to the World Health Organization (WHO) standard classification of anticancer drug adverse reactions. Survival curve was estimated by the Kaplan-Meier method. All $P$-values $\leq 0.05$ were considered statistically significant.

\section{Results}

\section{Patients and treatment compliance}

All patients agreed to receive TOMO during the recruitment. As shown in Table 1, the median age was 55 years. The median exposure dose for GTV was $67.5 \mathrm{~Gy}$, including $60 \mathrm{~Gy}$ for positive lymph nodes of upper neck and $54 \mathrm{~Gy}$ for positive lymph nodes of lower neck. Radiotherapy was suspended in ten patients (33\%) because of machine error and holidays. Cisplatin and bevacizumab were injected at doses of $130 \mathrm{mg}$ per cycle and $400 \mathrm{mg}$ every 2 weeks, respectively. The dosage of cisplatin was reduced in three patients $(6.7 \%)$ due to $>$ grade 3 mucosa reaction and myelosuppression (Table 2). After 3 months, 24 complete responses (80\%) and six partial responses $(20 \%)$ were observed as shown in Table 3.

Table I Characteristics of patients

\begin{tabular}{ll}
\hline Patient characteristics & Results \\
\hline Karnofsky Performance Scale & $\geq 90$ \\
Sex, n (\%) & $24(80 \%)$ \\
$\quad$ Male & $6(20 \%)$ \\
Female & $55(28-67)$ \\
$\quad$ Age (years), median (range) & \\
Histology (WHO), n (\%) & $12(40 \%)$ \\
$\quad$ Differentiated non-keratinizing carcinoma (type II) & $18(60 \%)$ \\
Undifferentiated non-keratinizing carcinoma (type III) & \\
Clinical stage, n (\%) & $19(63 \%)$ \\
III & $11(37 \%)$ \\
IVa & $0(0 \%)$ \\
IVb & \\
Tumor stage, n (\%) & $2(2 \%)$ \\
I & $3(20 \%)$ \\
2 & $18(54 \%)$ \\
3 & $7(24 \%)$ \\
4 & \\
Node stage, n (\%) & $3(10 \%)$ \\
0 & $17(57 \%)$ \\
I & $6(20 \%)$ \\
2 & $4(13 \%)$ \\
3 &
\end{tabular}

Abbreviation: WHO, World Health Organization.
Table 2 Acute toxic effects attributed to cisplatin and bevacizumab

\begin{tabular}{llll}
\hline Toxicity & \multicolumn{2}{l}{ Number of patients (\%) } \\
\cline { 2 - 4 } & Grade I-2 & Grade 3 & Grade 4 \\
\hline $\begin{array}{llll}\text { Hematological toxicities } \\
\text { Lymphopenia }\end{array}$ & $\mathrm{I}(3)$ & I7 (57) & $8(26)$ \\
$\begin{array}{l}\text { Leukopenia } \\
\text { Non-hematological toxicities }\end{array}$ & I4 (47) & $2(7)$ & 0 \\
$\quad$ Oropharyngeal mucositis & 2 I (70) & $3(10)$ & 0 \\
Pharyngalgia & $7(23)$ & I (3) & 0 \\
Diarrhea & $3(10)$ & 0 & 0 \\
Nausea & $27(90)$ & I (3) & 0 \\
Hypertension & 0 & 0 & 0 \\
Vomiting & $22(73)$ & 0 & 0 \\
Xerostomia & $24(80)$ & $3(10)$ & 0 \\
Headache & $3(10)$ & 0 & 0 \\
Fatigue & $26(87)$ & I (3) & 0 \\
ALT elevation & $4(13)$ & 0 & 0 \\
Infection & I (I0) & 0 & 0 \\
Weight loss & $7(23)$ & I (3) & 0 \\
\hline
\end{tabular}

Abbreviation: ALT, glutamic-pyruvic transaminase.

\section{Treatment toxicity}

Drug-related toxic effects were divided into two categories: hematological toxicity and non-hematological toxicity. Hematological toxicity was observed in all patients without serious infections. Leukopenia and lymphopenia were the most common grade 3-4 hematological toxicities. Twenty-four patients $(80 \%)$ experienced oropharyngeal mucositis which was the most common radiotherapy-related toxicity with high incidence. Twenty-six percent of patients experienced weight loss caused by pharyngeal pain and dysphagia resulting from oropharyngeal mucositis. Enteral and parenteral nutrition was essential for these patients. Symptoms gradually improved 1 month after radiotherapy. Nausea and vomiting was improved by symptomatic treatment. There was no creatinine elevation, hypertension, or proteinuria. All symptoms were relieved after 3 months, with the exception of $50 \%$ of patients reporting xerostomia, the commonest late-state toxicity.

\section{Discussion}

In this study, we have shown some evidence to support the fact that a combination of bevacizumab and concurrent chemoradiotherapy of cisplatin and TOMO was effective and safe for patients with NPC.

Table 3 Overall response rate (ORR)

\begin{tabular}{ll}
\hline Response (3 months after therapy) & Results, $\mathbf{n}(\%)$ \\
\hline Complete response & $24(80 \%)$ \\
Partial response & $6(20 \%)$ \\
Stable disease & 0 \\
Progressive disease & 0 \\
ORR & $30(100 \%)$ \\
\hline
\end{tabular}


The objective response rates of treatment with traditional chemotherapy drugs plus bevacizumab were $48 \%$ and $67 \%$ respectively. ${ }^{17}$ We evaluated curative effects 3 months after the treatment, the results showed that 24 patients reached complete response and six patients reached partial response. The overall response rate was $100 \%$. Our data suggest that the combination of cisplatin and bevacizumab regimen plus TOMO results in higher response rates.

In this process, all patients completed all courses of chemotherapy and targeted therapy. Moreover, in this research, treatment was completed with a lower incidence of interruption and dosage reduction than in many other researches. ${ }^{15}$ In a randomized Phase II study reported by Salama et al the patients were randomly assigned to receive FHX regimen (5-fluorouracil, hydroxyurea, and radiotherapy) or BFHX regimen (bevacizumab, 5-fluorouracil, hydroxyurea, and radiotherapy) in the treatment of head and neck cancer. Due to the higher local recurrence rate in the experimental group, the study was halted early among some of the patients. ${ }^{18}$ Furthermore, the results showed that the dosages of 5-fluorouracil and hydroxyurea in BFHX regimen should be reduced. ${ }^{18,19}$ Comparatively, in our research there was no evidence indicating that treatment interruption or dosage reduction would compromise the therapeutic efficacy.

In the previous studies of cisplatin-based treatment in locally advanced NPC, the treatment-related mortality was about $4 \% .{ }^{3-5,20}$ In the current study, no treatment-related death or severe toxic effects were observed. And there was no increase in the risk of death when the bevacizumab was added, which was consistent with other research of bevacizumab combined standard radiochemotherapy. ${ }^{21}$

In this study, the most common hematological toxic effect was leukopenia, the incidence was 54\%. While in other cisplatin-based researches the incidence of leukopenia ranged from $40 \%$ to $47 \% .{ }^{4,5,10}$ Due to the small sample size in this research, we did not observe any increase in the risk of hematological toxicity among those patients who were treated with high-dose cisplatin and bevacizumab plus TOMO. The main non-hematological toxicity observed in this research included radiotherapy-related acute skin and mucosal toxic effects. Acute skin and mucosal toxic effects were reversible and curable. The morbidity rate of oropharyngeal mucositis $>$ grade 3 was about $24 \%$ in this study (Figure 1). Historically, the incidence of grade 3-4 oral mucositis among patients who received concurrent cetuximab and radiotherapy, reported by Pfister et al and Argiris et al was 38\% and 54\%, respectively. 22,23 Another Phase III study from People's Republic of China showed that the grade 3-4 mucositis rate was $48 \%$ in the patients treated with concomitant cisplatin and 2D-radiotherapy. ${ }^{24}$

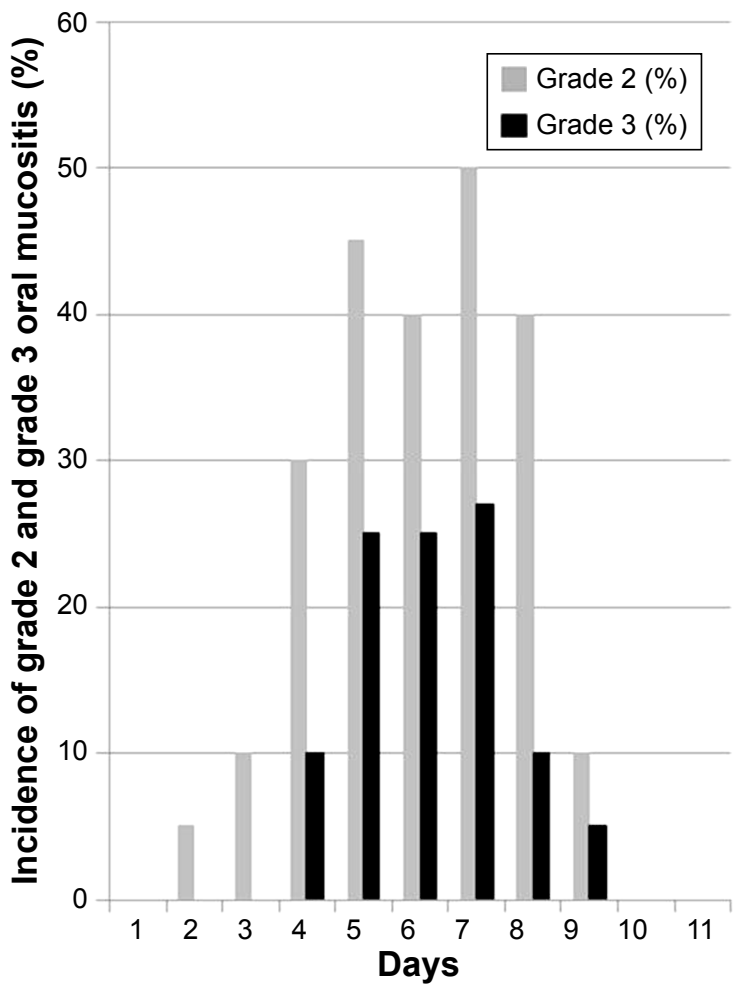

Figure I Incidence of grade 2 and grade 3 oral mucositis in our research. Note: We recorded the mucositis cases everyday, from day I to day II.

We also showed that xerostomia is one of the most common but well-controlled side effects with relatively low severity here in this research, which was similar to some other researches based on 3D-radiotherapy and intensity-modulated radiation therapy. ${ }^{25,26}$ This may be explained by the short treatment time of TOMO, protection of parotid glands or heterogeneous samples. More importantly, in this study, there was not any new type of side effect or any higher grade side effects relative to other similar studies.

There is no strict patient eligibility when measuring VEGF mutation because the over expression of VEGF $(>80 \%)$ in NPC has been well recognized already. In previous clinical trials, Johnson et al had proven that bevacizumab increased the risk of bleeding in squamous cell carcinoma patients. ${ }^{27} \mathrm{But}$ in many Phase II clinical trials of head and neck cancers, bevacizumab has been safely used with chemotherapy, radiotherapy, or targeted therapy with very few cases of bleeding. ${ }^{14,21,28}$ Here, we used strict eligibility and exclusion criteria, which may explain why no bleeding cases were observed in this research. Therefore, we believe that it is still helpful to select suitable patients for treatment of bevacizumab in NPC.

Despite the small sample size in this clinical trial, the results revealed that bevacizumab plus cisplatin and TOMO was feasible and useful in treatment of locally advanced NPC. The side effects and toxicities were similar to other 
researches and acceptable. Our next work is a long-term follow-up of these patients.

\section{Acknowledgment}

Grant support: The Wu Jieping Foundation. The costs of experimentation and publication of this article were all defrayed from this foundation.

\section{Disclosure}

The authors have no conflicts of interest to disclose.

\section{References}

1. Lee AW, Sze WM, Au JS, et al. Treatment results for nasopharyngeal carcinoma in the modern era: the Hong Kong experience. Int $J$ Radiat Oncol Biol Phys. 2005;61(4):1107-1116.

2. Browman GP, Hodson DI, Mackenzie RJ, et al. Choosing a concomitant chemotherapy and radiotherapy regimen for squamous cell head and neck cancer: A systematic review of the published literature with subgroup analysis. Head Neck. 2001;23(7):579-589.

3. Marcial VA, Pajak TF, Mohiuddin M, et al. Concomitant cisplatin chemotherapy and radiotherapy in advanced mucosal squamous cell carcinoma of the head and neck. Long-term results of the Radiation Therapy Oncology Group study 81-17. Cancer. 1990;66(9):1861-1868.

4. Forastiere AA, Goepfert H, Maor M, et al. Concurrent chemotherapy and radiotherapy for organ preservation in advanced laryngeal cancer. N Engl J Med. 2003;349(22):2091-2098.

5. Adelstein DJ, Li Y, Adams GL, et al. An intergroup phase III comparison of standard radiation therapy and two schedules of concurrent chemoradiotherapy in patients with unresectable squamous cell head and neck cancer. J Clin Oncol. 2003;21(1):92-98.

6. Kesmezacar H, Ayhan E, Unlu MC, Seker A, Karaca S. Predictors of mortality in elderly patients with an intertrochanteric or a femoral neck fracture. J Trauma. 2010;68(1):153-158, doi:10.1097/ TA.0b013e31819adc50 (2010).

7. National Comprehensive Cancer Network: About NCCN; NCCN Guidelines ${ }^{\circledR}$. Available from: http://www.nccn.org/professionals/physicians_gls/pdf/head-and-neck. Accessed May 25, 2015.

8. Fidler IJ. Cancer metastasis. Br Med Bull. 1991;47(1):157-177.

9. Fakhrejahani E, Toi M. Antiangiogenesis therapy for breast cancer: an update and perspectives from clinical trials. Jpn J Clin Oncol. 2014; 44(3):197-207.

10. Kirstein MM, Lange A, Prenzler A, Manns MP, Kubicka S, Vogel A. Targeted Therapies in Metastatic Colorectal Cancer: A Systematic Review and Assessment of Currently Available Data. Oncologist. 2014;19(11):1156-1168.

11. Lai GG, Penson RT. Bevacizumab and ovarian cancer. Drugs Today (Barc). 2011;47(9):669-681.

12. Hsu HW, Wall NR, Hsueh CT, et al. Combination antiangiogenic therapy and radiation in head and neck cancers. Oral oncol. 2014;50(1):19-26.

13. Kyzas PA, Cunha IW, Ioannidis JP. Prognostic significance of vascular endothelial growth factor immunohistochemical expression in head and neck squamous cell carcinoma: a meta-analysis. Clin Cancer Res. 2005;11(4):1434-1440.

OncoTargets and Therapy

\section{Publish your work in this journal}

OncoTargets and Therapy is an international, peer-reviewed, open access journal focusing on the pathological basis of all cancers, potential targets for therapy and treatment protocols employed to improve the management of cancer patients. The journal also focuses on the impact of management programs and new therapeutic agents and protocols on

Submit your manuscript here: http://www.dovepress.com/oncotargets-and-therapy-journal
14. Fury MG, Lee NY, Sherman E, et al. A phase 2 study of bevacizumab with cisplatin plus intensity-modulated radiation therapy for stage III/IVB head and neck squamous cell cancer. Cancer. 2012;118(20):5008-5014.

15. Jain RK. Normalization of tumor vasculature: an emerging concept in antiangiogenic therapy. Science. 2005;307(5706):58-62.

16. Ma BB, Kam MK, Leung SF, etal. A phase II study of concurrent cetuximabcisplatin and intensity-modulated radiotherapy in locoregionally advanced nasopharyngeal carcinoma. Ann Oncol. 2012;23(5):1287-1292.

17. Aravantinos G, Pectasides D. Bevacizumab in combination with chemotherapy for the treatment of advanced ovarian cancer: a systematic review. J Ovarian Res. 2014;7:57.

18. Salama JK, Haraf DJ, Stenson KM, et al. A randomized phase II study of 5-fluorouracil, hydroxyurea, and twice-daily radiotherapy compared with bevacizumab plus 5-fluorouracil, hydroxyurea, and twice-daily radiotherapy for intermediate-stage and T4N0-1 head and neck cancers. Ann Oncol. 2011;22(10):2304-2309.

19. Seiwert TY, Haraf DJ, Cohen EE, et al. Phase I study of bevacizumab added to fluorouracil- and hydroxyurea-based concomitant chemoradiotherapy for poor-prognosis head and neck cancer. J Clin Oncol. 2008;26(10):1732-1741.

20. Garden AS, Harris J, Vokes EE, et al. Preliminary results of Radiation Therapy Oncology Group 97-03: a randomized phase ii trial of concurrent radiation and chemotherapy for advanced squamous cell carcinomas of the head and neck. J Clin Oncol. 2004;22(14):2856-2864.

21. Lee NY, Zhang Q, Pfister DG, et al. Addition of bevacizumab to standard chemoradiation for locoregionally advanced nasopharyngeal carcinoma (RTOG 0615): a phase 2 multi-institutional trial. Lancet Oncol. 2012;13(2):172-180.

22. Pfister DG, Su YB, Kraus DH, et al. Concurrent cetuximab, cisplatin, and concomitant boost radiotherapy for locoregionally advanced, squamous cell head and neck cancer: a pilot phase II study of a new combined-modality paradigm. J Clin Oncol. 2006;24(7):1072-1078.

23. Argiris A, Heron DE, Smith RP, et al. Induction docetaxel, cisplatin, and cetuximab followed by concurrent radiotherapy, cisplatin, and cetuximab and maintenance cetuximab in patients with locally advanced head and neck cancer. J Clin Oncol. 2010;28(36):5294-5300.

24. Chan AT, Teo PM, Ngan RK, et al. Concurrent chemotherapyradiotherapy compared with radiotherapy alone in locoregionally advanced nasopharyngeal carcinoma: progression-free survival analysis of a phase III randomized trial. J Clin Oncol. 2002;20(8):2038-2044.

25. Kam MK, Teo PM, Chau RM, et al. Treatment of nasopharyngeal carcinoma with intensity-modulated radiotherapy: the Hong Kong experience. Int J Radiat Oncol Biol Phys. 2004;60(5):1440-1450.

26. Kwong DL, Pow EH, Sham JS, et al. Intensity-modulated radiotherapy for early-stage nasopharyngeal carcinoma: a prospective study on disease control and preservation of salivary function. Cancer. 2004;101(7): $1584-1593$.

27. Johnson DH, Fehrenbacher L, Novotny, WF, et al. Randomized phase II trial comparing bevacizumab plus carboplatin and paclitaxel with carboplatin and paclitaxel alone in previously untreated locally advanced or metastatic non-small-cell lung cancer. J Clin Oncol. 2004;22(11):2184-2191.

28. Argiris A, Kotsakis AP, Hoang T, et al. Cetuximab and bevacizumab: preclinical data and phase II trial in recurrent or metastatic squamous cell carcinoma of the head and neck. Ann Oncol. 2013;24(1):220-225.

\section{Dovepress}

patient perspectives such as quality of life, adherence and satisfaction. The manuscript management system is completely online and includes a very quick and fair peer-review system, which is all easy to use. Visit http://www.dovepress.com/testimonials.php to read real quotes from published authors. 